Bond University

Research Repository

\title{
Ad libitum Mediterranean diet reduces subcutaneous but not visceral fat in patients with coronary heart disease: A randomised controlled pilot study
}

Mayr, Hannah; Itsiopoulos, Catherine; Tierney, Audrey C; Kucianski, Teagan; Radcliffe, Jessica; Garg, Manohar; Willcox, Jane C.; Thomas, Colleen J

Published in:

Clinical Nutrition ESPEN

DOI:

10.1016/j.clnesp.2019.05.001

Licence:

CC BY-NC-ND

Link to output in Bond University research repository.

Recommended citation(APA):

Mayr, H., Itsiopoulos, C., Tierney, A. C., Kucianski, T., Radcliffe, J., Garg, M., Willcox, J. C., \& Thomas, C. J. (2019). Ad libitum Mediterranean diet reduces subcutaneous but not visceral fat in patients with coronary heart disease: A randomised controlled pilot study. Clinical Nutrition ESPEN, 32(2019), 61-69.

https://doi.org/10.1016/j.clnesp.2019.05.001

\section{General rights}

Copyright and moral rights for the publications made accessible in the public portal are retained by the authors and/or other copyright owners and it is a condition of accessing publications that users recognise and abide by the legal requirements associated with these rights.

For more information, or if you believe that this document breaches copyright, please contact the Bond University research repository coordinator. 
1 Ad libitum Mediterranean diet reduces subcutaneous but not visceral fat in patients with coronary heart disease: a randomised controlled pilot study

4 Hannah L Mayr $\mathrm{PhD}^{1,2,3} *$, Catherine Itsiopoulos $\mathrm{PhD}^{1}$, Audrey C Tierney $\mathrm{PhD}^{4,5}$, Teagan Kucianski MDiet 5 Jessica Radcliffe $\mathrm{PhD}^{5}$, Manohar Garg $\mathrm{PhD}^{6}$, Jane Willcox $\mathrm{PhD}^{1}$ and Colleen $\mathrm{J}^{\mathrm{Th}}$ homas $\mathrm{PhD}^{7}$

6 ' ${ }^{1}$ School of Allied Health, Human Services and Sport, La Trobe University, Melbourne, Victoria, 3086,

7 Australia

$8 \quad{ }^{2}$ Nutrition and Dietetics Department, Princess Alexandra Hospital, Brisbane, Queensland, 4102, Australia

$9 \quad{ }^{3}$ Bond University Nutrition and Dietetics Research Group, Faculty of Health Sciences and Medicine, Bond

10 University, Gold Coast, Queensland, 4226, Australia

$11{ }^{4}$ School of Allied Health, University of Limerick, Castletroy, Limerick, V94 T9PX, Ireland

$12{ }^{5}$ Department of Dietetics, Nutrition and Sport, School of Allied Health, Human Services and Sport, La Trobe 13 University, Melbourne, Victoria, 3086, Australia

$14{ }^{6}$ Nutraceuticals Research Program, School of Biomedical Sciences \& Pharmacy, University of Newcastle, 15 New South Wales, 2308, Australia

$16{ }^{7}$ Department of Physiology, Anatomy and Microbiology, School of Life Sciences, La Trobe University, 17 Melbourne, Victoria, 3086, Australia

*Correspondence: Dr Hannah L Mayr

E: H.Mayr@1atrobe.edu.au; Tel.: +61-7-3176 7938.

21 Postal Address: Ground floor Building 15, Nutrition and Dietetics Department, Princess Alexandra Hospital, 22 Woolloongabba, Queensland, 4102, Australia. 
Abstract

Background \& aims: The Mediterranean diet (MedDiet) is recognised to reduce risk of coronary heart disease

30 (CHD), in part, via its anti-inflammatory and antioxidant properties, which may be mediated via effects on

31 body fat distribution. Diet efficacy via these mechanisms is however unclear in patients with diagnosed CHD.

32 This study aimed to determine: (1) the effect of ad libitum MedDiet versus low-fat diet intervention on adiposity, anti-inflammatory marker adiponectin, oxidative stress marker malondialdehyde (MDA) and traditional CVD risk markers, and (2) whether improvement in MedDiet adherence score in the pooled cohort was associated with these risk markers, in a pilot cohort of Australian patients post coronary event.

Methods: Participants (62 \pm 9 years, $83 \%$ male) were randomised to 6-month ad libitum MedDiet ( $n=34)$ or low-fat diet $(n=31)$. Pre- and post-intervention, dietary adherence, anthropometry, body composition (Dualenergy X-ray Absorptiometry) and venepuncture measures were conducted.

Results: The MedDiet group reduced subcutaneous adipose tissue (SAT) area compared to the low-fat diet group $\left(12.5 \mathrm{~cm}^{2}\right.$ more, $\left.p=0.04\right)$ but not visceral adipose tissue or other body composition measures. In the pooled cohort, participants with greatest improvement in MedDiet adherence score had significantly lower waist circumference $(-2.81 \mathrm{~cm}, p=0.01)$ and SAT area $\left(-27.1 \mathrm{~cm}^{2}, \mathrm{p}=0.04\right)$ compared to participants with no improvement in score at 6-months. There were no changes in adiponectin, MDA or other risk markers in the MedDiet compared to low-fat diet group, and no differences in 6-month levels between categories of improvement in MedDiet score $(p>0.05)$. Within the MedDiet group only, the proportion of participants taking beta-blocker medication reduced from baseline to 6 -months ( $71 \%$ vs. $56 \%, p$-trend $=0.007)$.

Conclusions: Adherence to 6-month ad libitum MedDiet reduced subcutaneous fat and waist circumference which discounts the misconception that this healthy but high fat diet leads to body fat gain. The effect of MedDiet on body fat distribution and consequent anti-inflammatory and antioxidant effects, as well as need for medications, in patients with CHD warrants exploration in larger studies. Clinically significant effects on these markers may require adjunct exercise and/or caloric restriction.

Trial registration: ACTRN12616000156482. composition 


\section{Introduction}

The Mediterranean diet (MedDiet) pattern has a strong scientific evidence base for reducing risk of coronary heart disease (CHD) and adverse cardiovascular disease (CVD) events $(1,2)$. Nonetheless, the majority of studies investigating the MedDiet have been conducted in Mediterranean countries. A low-fat diet was the standard care recommendation for prevention and treatment of CHD in Australia for many years (3), however, a recent position statement from the National Heart Foundation of Australia promotes a variety of healthy dietary patterns, rather than focusing on isolated nutrients, for cardiovascular health (4).

Atherosclerosis is the underlying pathology responsible for CHD. Derangements in lipid levels, blood pressure and insulin homeostasis each lead to endothelial dysfunction, which plays a pivotal role in initiating the atherosclerotic process (5). A number of studies, including in the Australian setting, have demonstrated that the MedDiet improves CVD risk factors, including improvements in triglycerides and high-density lipoprotein (HDL) cholesterol, blood pressure, glucose metabolism and reduced risk of type 2 diabetes mellitus (T2DM) (6-13). These studies were conducted in patients at risk of, but without, established CHD. In CHD, especially in those who have suffered acute coronary syndrome (ACS), pharmacotherapy is used to achieve recommended lipid, glucose and blood pressure targets (14), hence the possibility to attain additional impact of diet on these risk factors may not be observed in these patients. In fact, the limited published data on the impact of MedDiet on secondary prevention of ACS demonstrated that the diet may be operating independently of traditional CVD risk factors (1).

Atherosclerosis is recognised to be an inflammatory condition, which is related to both the chronic development of plaque and its acute rupture (15). In addition, obesity, especially increased visceral fat, is causally linked to chronic low-grade inflammation $(16,17)$. In an obese state, adipose tissue generates pro-

81 inflammatory adipokines, including interleukin-6 (IL-6), whereas anti-inflammatory adipokines, including adiponectin, are down-regulated (18). High serum concentrations of adiponectin are associated with decreased risk of CHD $(19,20)$. Oxidative stress has also been recognised to increase risk of cardiovascular events in 
patients with CHD, through increased oxidation of LDL particles and endothelial dysfunction (21). Plasma malondialdehyde (MDA), a non-invasive measure of lipid peroxidation, is a recognised marker of oxidative stress and elevated MDA levels are reported in patients with CHD (22).

To better understand how dietary interventions moderate CHD risk, it is important to ascertain their effect on novel markers such as adiposity, inflammation and oxidative stress in addition to classic cardiometabolic risk markers. A recent review of intervention trials demonstrated that the MedDiet can reduce central obesity; however, most studies measured waist circumference without distinguishing visceral and subcutaneous fat and included patients without CHD (23). Meta-analyses of randomised controlled trials (RCTs) have also concluded that intervention with the MedDiet improves a range of established inflammatory and oxidative stress markers $(24,25)$. However, a recent systematic review of the literature established that no studies have investigated the effect of MedDiet on adiponectin in patients with diagnosed CHD (26). Moreover, with respect to lipid peroxidation, MedDiet intervention improved MDA levels in patients at risk of but without CHD (27).

We have previously reported results from this pilot MedDiet intervention, showing no improvement in the inflammatory markers high sensitivity C-Reactive Protein (hs-CRP) or hs-IL-6, despite significant improvement in MedDiet adherence and dietary anti-inflammatory potential (measured by the dietary inflammatory index) in Australian patients who have experienced an ACS event $(28,29)$. Therefore, the primary aim of the present analysis was to determine the effect of ad libitum MedDiet versus low-fat diet intervention on additional cardiometabolic risk markers, including compartmental adiposity, antiinflammatory marker adiponectin, and MDA levels in the same pilot cohort. A secondary aim was to determine whether improvement in MedDiet adherence score in the pooled cohort was associated with resultant improvement in risk marker levels. Results from this pilot will be used to inform feasibility and sample size requirements for future analyses.

\section{Materials and Methods}


112 The data reported in this study was collected in the pilot of the AUStralian MEDiterranean Diet Heart Trial 113 (AUSMED Heart Trial), a multi-centre, parallel design, randomised controlled trial (RCT) of 6-month 114 MedDiet versus low-fat diet intervention for the secondary prevention of CHD at 12-months in a multi-ethnic 115 Australian population (Australia and New Zealand Clinical Trials Register: ACTRN12616000156482) (30). 116 As noted above, this pilot study and methodology, including results for nutritional intake and diet quality (29), 117 the dietary inflammatory index, hs-CRP and hs-IL-6 $(28,31)$ has previously been reported.

\subsection{Recruitment of CHD Patients}

120 Patients for this pilot study were recruited from two tertiary hospitals in Melbourne, Australia between October 1212014 and November 2016. Eligible patients were adults with CHD, able to read and write in English and who 122 had experienced ACS defined as at least one of the following: acute myocardial infarction (AMI); angina 123 pectoris with documented coronary artery disease on imaging; coronary artery bypass grafting; or percutaneous coronary intervention. The study was conducted in accordance with the Declaration of Helsinki (32) and the CONSORT guidelines (33). All procedures involving patients were approved by the Human Research Ethics Committees of The Northern Hospital, St Vincent's Hospital Melbourne, and La Trobe University, with written informed consent obtained from all enrolled participants before randomisation.

\subsection{Randomisation of Participants and Diet Interventions}

130 At a pre-baseline appointment, enrolled participants were randomly assigned in a 1:1 ratio to the MedDiet 131 group or the low-fat diet group using a stratified approach (based on sex, age and prior AMI). Baseline, 3- and 132 6-month face-to-face appointments were conducted to obtain dietary data and for counselling with the dietitian. 133 Five short phone reviews for follow-up dietary counselling with the dietitian also occurred across the 6-months, 134 at weeks 3, 6, and 9 and months 4 and 5. Both diets were prescribed ad libitum with no specific 135 recommendations on energy restriction. All participants continued to receive standard medical care provided 136 at their respective hospital or primary care settings and their access to outside health services during the study 137 intervention period was recorded at each appointment. 
The rationale, development and resources provided with our MedDiet intervention, designed for use in chronic

141 disease intervention trials in the Australian setting $(30,34)$, has been explained and published in detail 142 elsewhere (35). Briefly, the diet was modelled via a 2-week meal plan which incorporated key dietary 143 components of a MedDiet and a mix of traditional and modified recipes considered to be realistic options in 144 the multi-ethnic Australian setting. Food group recommendations included: daily intake of extra virgin olive oil (EVOO), wholegrain cereals, vegetables, fruit and nuts; regular intake of fish and seafood, legumes and yoghurt; and limited intake of commercial sweets or pastries and red or processed meat. Poultry, eggs and feta cheese were recommended in moderation. For existing alcohol drinkers, red wine was suggested to be consumed in moderation (1-2 standard glasses) with meals. To facilitate dietary compliance and to encourage intake of staple Mediterranean foods less familiar to this Australian population, a hamper was provided to participants at baseline and 3-months, including $6 \mathrm{~L} \mathrm{EVOO} \mathrm{(to} \mathrm{achieve} 60-80 \mathrm{~mL} /$ day) and $1.2 \mathrm{~kg}$ nuts (almonds, walnuts and hazelnuts to achieve $30 \mathrm{~g} / \mathrm{day})$.

\subsubsection{Low-fat Diet}

154 Participants in the low-fat diet group were instructed to follow the standard diet recommendations provided to cardiac patients in Australia at the time this study was developed (in 2014). Recommendations from the National Heart Foundation (3) and Australian Dietary Guidelines $(36,37)$ were consulted for design of the low-fat diet. Food group recommendations included daily intake of grains and cereals (mostly whole grains), vegetables, lean meats and alternatives, fruit, and low-fat dairy foods (36). Participants were provided with a supermarket voucher at each of their three face-to-face appointments to aid compliance and encourage continuation in the trial.

\subsection{Study Measures}

163 This study reports on outcome measurements collected at the baseline and 6-month appointments. Data on 164 medical conditions was collected from medical records and in consultation with hospital staff during the screening process, and via a questionnaire at the pre-baseline appointment. Participants completed a self-report 
characteristics, including medication and supplement use. A modified version of the survey was completed at both 3- and 6-month appointments, which re-assessed lifestyle and clinical characteristics.

\subsubsection{Dietary Intake}

The week prior to each face-to-face appointment the participants completed a 7-day food diary which was entered into FoodWorks (Version 8, Xyris software Australia Pty Ltd) for nutrient and food group intake analyses. The 14-point Mediterranean Diet Adherence Screener (MEDAS), generated and validated for the PREDIMED study (38), was measured at each appointment for both diet study groups.

\subsubsection{Cardiometabolic Risk Markers}

Our methods for assessment of activity levels, anthropometry, body composition, blood pressure and pathology measures have also been described previously (31). Increased physical activity was not a target of this intervention, however, physical activity levels were assessed to account for any potential confounding effects on outcome markers. Participants wore a triaxial Actigraph accelerometer (WGT3X-BT; Actigraph Corp, Florida, United States) for one week prior to their appointments. Established criteria (39) were used to determine time spent as min /week in moderate-to-vigorous physical activity (MVPA) or as sedentary time. Weight, height and waist circumference measures were performed according to the International Society for the Advancement of Kinanthropometry (ISAK) standards for anthropometric assessment (40). Whole body composition was measured using a fan beam densitometer Dual-energy X-ray Absorptiometry (DXA) machine (Hologic, Discovery W, USA), with analysis performed using QDR ${ }^{\mathrm{TM}}$ (Quantitative Digital Radiography) for Windows. Measurements obtained from each scan were total body lean and fat mass, total body and regional fat percentage, subcutaneous adipose tissue (SAT) and visceral adipose tissue (VAT) areas. Hologic scientists developed their method for measuring VAT from DXA (41), which is highly correlated ( $\mathrm{r}=0.93)$ and linearly related to VAT measurements by computed tomography (42). Fat mass index (FMI) was calculated by dividing the total body fat mass $(\mathrm{kg})$ by height $(\mathrm{m})$ squared (43). Systolic blood pressure (SBP), diastolic blood pressure (DBP) and heart rate (HR) were measured using an automated blood pressure monitor (OMRON Tp9, IntellisenSense, Australia). Hypertension (presence or history of) was classified based on whether the participants were prescribed medication with anti-hypertensive effect (angiotensin converting enzyme [ACE] 
inhibitor, angiotensin 2 receptor blocker, Beta $[\beta]$-blocker or $\mathrm{Ca}^{2+}$ channel blocker) and/or mean baseline blood pressure reading of $\mathrm{SBP}>140 \mathrm{mmHg}$ or $\mathrm{DBP}>90 \mathrm{mmHg}$ (44). Diagnosis of T2DM was determined by consulting participant medical history records.

Fasting blood samples were taken by venepuncture and processed immediately into serum/plasma aliquots (as published in detail elsewhere (45)) which were stored at $-80^{\circ} \mathrm{C}$ until laboratory assays were conducted. Serum low-density lipoprotein (LDL) cholesterol, HDL cholesterol, triglycerides and hs-CRP levels were measured at a commercial laboratory (Dorevitch Pathology Pty Ltd, Heidelberg, Australia). Other biomarker measures were performed by trained personnel at La Trobe University, except for MDA which was performed at the University of Newcastle. Enzyme-linked immunosorbent assay (ELISA) kits were used to measure serum hsIL-6 levels (Abcam Australia Pty Ltd, \#ab46042), serum adiponectin levels (Invitrogen, Thermofisher Scientific, \#KHP0041) and plasma MDA levels (Abcam Australia Pty Ltd, \#ab1 18970). Fasting serum glucose levels were measured using the enzymatic hexokinase method by a chemical analyser (Indiko, Thermofisher Scientific). Personnel performing laboratory analyses were blinded to study group allocation of samples.

\subsection{Statistical Analyses}

This study represented a preliminary analysis in a pilot cohort and therefore a sample size calculation was not performed prior to conducting the measures (46). The broader AUSMED Heart Trial is powered to detect a significant effect of MedDiet on secondary cardiovascular endpoints and will recruit 1000 participants (30). All statistical analyses were conducted in $\operatorname{SPSS}^{\circledR}$ statistical package version 25 (IBM Corp, released 2015). Statistical significance was set at $p<0.05$. Data are presented as means \pm standard deviation (SD) or standard error (SEM), medians (interquartile range [IQR]) or $\mathrm{n}(\%)$, as appropriate. The Kolmogorov-Smirnov test was applied to assess the normality of continuous variables. According to this, an Independent Student's $t$-test or non-parametric Mann-Whitney U test was used to compare continuous variables. Categorical variables were compared using the Chi-square test.

All outcome measures were analysed based on intention-to-treat with missing data included by bringing baseline or 3-month observations forward, assuming no change (47). Cochran's Q test assessed changes in the 
proportion of participants taking medication and supplement classes from baseline to 3- and 6-months within each study group. Repeated measures ANOVA (analysis of variance) assessed changes in cardiometabolic risk marker variables from baseline to 6-months between groups. Measures which were non-parametric were log transformed to improve their distribution. The main ANOVA results assessed for effect were (1) group (significant change in one study group compared to the other) and (2) time (significant change in pooled study groups). Post-hoc tests were performed to determine within-group changes (Paired Samples $t$-test). Analyses for all risk markers were adjusted for change in MVPA and haemodynamic and pathology measures were additionally adjusted for baseline BMI. The repeated measures analysis inherently controlled for participant characteristics not subject to change and were not different between study groups at baseline, including sex and T2DM status. The between-group findings for adiponectin and MDA levels were used to perform a sample size calculation to inform future analyses (described in Results).

To account for any cross-over in improvement towards the MedDiet pattern in participants of the low-fat diet group, analyses were also performed in the pooled cohort (with inclusion of hs-CRP and hs-IL-6 which have not previously been analysed in this way). Tertiles of change in participant MEDAS scores from baseline to 6-months were created in SPSS. Least-squared means (95\% confidence interval $[\mathrm{CI}])$ of cardiometabolic risk markers at 6-months were estimated across the tertiles of MEDAS change. Multi-variable general linear models adjusted for baseline value, sex, age, T2DM, time since coronary event and change in MVPA were used to estimate the differences in means across tertiles. For hs-CRP, participants with serum levels $>10 \mathrm{mg} / \mathrm{L}$ were excluded from analyses, as these higher concentrations reflect acute rather than chronic inflammation (48).

\section{Results}

\subsection{Participants}

248 Randomisation to diet study groups, completion of study appointments and number and reasons for withdrawal have been reported elsewhere $(28,29)$. Briefly, of 73 randomised participants, 65 attended a baseline appointment and started the intervention. The subsequent attrition rate was $14 \%$, with 2 and 7 participants 
251 dropping out from the low-fat diet and MedDiet groups respectively. Participants were lost to follow up ( $\mathrm{n}=2$ ) 252 or discontinued due to relocation $(n=2)$, non-cardiac medical problems $(n=3)$ or family related issues $(n=2)$.

253 There were no significant differences for sociodemographic or clinical characteristics between those 254 participants that dropped out compared to completers.

256 As reported in Table 1, the cohort represented a mostly male, middle to late aged group of which close to half 257 were born outside Australia (18\% were born in the Mediterranean region). Participants had highly variable 258 levels of MVPA (total range 3 to $665 \mathrm{~min} /$ week), their baseline MedDiet adherence was low (mean MEDAS 259 score of 5 out of 14) and $80 \%$ had previously attended a cardiac rehabilitation program. Most participants had 260 experienced an AMI and undergone percutaneous coronary intervention with a median time since ACS event 261 of $<6$ months prior. Close to one third had diagnosed T2DM and nearly all had current or previous 262 hypertension. Participants were prescribed multiple medications, of which anti-platelets and statins (both $263>90 \%$ ) were the most common (Supplementary Materials, Table S1). Close to half the participants were taking 264 nutrition supplements, of which vitamin D (19\%) and omega-3 (15\%) were the most common (Table S1). 265 There were no significant differences at baseline between the diet study groups for any of these reported 266 sociodemographic, lifestyle or clinical characteristics.

268 Table 1. Participant baseline characteristics in the study groups

\begin{tabular}{lll}
\hline Characteristic & Low-fat $(\boldsymbol{n}=\mathbf{3 1})$ & MedDiet $(\boldsymbol{n}=\mathbf{3 4})$ \\
\hline Sociodemographic & & \\
Male & $27(87.1)$ & $27(79.4)$ \\
Age (years) & $61.8 \pm 9.5$ & $61.8 \pm 9.2$ \\
Country of birth & & \\
$\quad$ Australia & $18(58.1)$ & $20(58.8)$ \\
$\quad$ Other & $13(41.9)$ & $14(41.2)$ \\
$\quad$ Mediterranean country & $7(22.6)$ & $5(14.7)$ \\
Lifestyle & & \\
Current smoker & $3(9.7)$ & $6(18.2)$
\end{tabular}




$\begin{array}{lll}>100 \text { cigarettes in lifetime } & 18(58.1) & 20(58.8) \\ \text { BMI }\left(\mathrm{kg} / \mathrm{m}^{2}\right) & 29.1 \pm 5.3 & 30.7 \pm 5.0 \\ \text { MVPA (min /week) } \dagger & 120.0(189.5) & 153.0(210.0) \\ \text { MEDAS (score out of } 14) & 4.8 \pm 1.8 & 5.6 \pm 2.2 \\ \text { Cardiac rehabilitation } & 26(83.9) & 25(73.5) \\ \text { Medical History } & & \\ \text { Acute myocardial infarction } & 22(71.0) & 23(67.6) \\ \text { Percutaneous coronary intervention } & 25(80.6) & 25(73.5) \\ \text { Coronary artery bypass grafting } & 8(25.8) & 7(20.6) \\ \text { Time since event (months) } \dagger & 4.5(6.5) & 5.1(15.2) \\ \text { Type 2 diabetes mellitus } & 9(29.0) & 10(29.4) \\ \text { Hypertension } & 31(100) & 31(91.2) \\ \text { Depression (diagnosed) } & 4(12.9) & 6(17.6)\end{array}$

Values are n (\%), Mean \pm SD or Median (IQR)†. MedDiet; Mediterranean diet; BMI, body mass index; MVPA, moderate-to-vigorous physical activity; MEDAS, Mediterranean diet adherence screener.

There were no significant differences between the groups for frequency of attendance at each of the study appointments and phone call reviews conducted across the diet intervention period (Supplementary Material, Table S2). The proportion of participants who attended each of the appointments or reviews was $80 \%$ or more.

275 The participants reported having accessed a variety of other health services during the intervention period, but 276 there were no significant differences between the study groups (Table S2). There was a reduction in the 277 proportion of participants prescribed $\beta$-blockers in the MedDiet group between baseline and at 3-months (from 27824 to 19 participants) and this was maintained at 6-months ( $p$-trend=0.007). There were no other changes in 279 the proportion of participants taking prescribed medications in either study group (Table S1). Participants 280 reported high medication compliance at baseline and this remained consistent throughout the study. There were 281 no significant changes within either study group for use of nutrition supplements across the intervention period 282 (Table S1). 
Daily intake of food group serves, energy and nutrients have been reported previously (29). Briefly, in the MedDiet group, in line with recommendations, consumption of olive oil, fruit, yoghurt, nuts, legumes and seafood significantly increased, whereas red and processed meats decreased after 6-months. There were no significant changes for dietary intake of individuals nutrients or foods in the low-fat diet group. There was a significantly greater improvement in mean MEDAS score in the MedDiet group $(+4.8$ points from a baseline score of 5.6 out of 14) compared to low-fat diet group $(+1.2$ points from a baseline of 4.8 out of 14$)(p<0.001)$. The small improvement in MedDiet score in the low-fat diet participants was related to their improved adherence to score components for vegetable intake and use of butter/cream. There was no significant difference for change in MEDAS score between participants born in a Mediterranean country versus not, as assessed separately within the study groups and with the study groups pooled. No participants reported harmful side effects or adverse events directly related to the dietary interventions.

\subsection{Activity Levels}

There were no significant changes in time spent as sedentary or in MVPA activity between baseline and 6months in the MedDiet or low-fat diet groups (Table 2, all variables reported as mean $\pm \mathrm{SEM}$ ). However, there was a high level of individual variability for these measures and participants in the MedDiet group tended to reduce their MVPA level (by $42 \mathrm{~min} /$ week, $p=0.20$ ), hence this justified controlling for any activity changes in the risk marker analyses.

\subsection{Anthropometry and Body Composition}

With regards to anthropometry and body composition measures, there was a significant between-group difference for 6-month change in SAT area only $\left(-12.1 \pm 6.5 \mathrm{~cm}^{2}\right.$ in the MedDiet vs. $+0.4 \pm 6.9 \mathrm{~cm}^{2}$ in the lowfat diet group, $p=0.04$; Figure 1). VAT area did not change within the MedDiet $\left(-0.1 \pm 3.8 \mathrm{~cm}^{2}\right)$ or the low-fat

$\operatorname{diet}\left(+2.4 \pm 4.0 \mathrm{~cm}^{2}\right)$ groups $(p=0.58$, Figure 1). There were no significant within-group changes for weight, BMI, waist circumference, or waist-hip ratio (Table 2). There was no significant reduction in waist circumference over time in the pooled study groups $(-1.1 \mathrm{~cm}, p=0.07$ within the MedDiet and $-0.4 \mathrm{~cm}, p=0.52$ within in the low-fat diet group). There was also no significant reduction in total body fat $\%$ over time in the pooled study groups $(-0.6 \%, p=0.06$ within the MedDiet and $-0.4 \%, p=0.23$ within in the low-fat diet group). 
313 Leg fat $\%$, however, decreased significantly over time in the pooled study groups $(p=0.04)$ with a significant 314 reduction within the MedDiet group $(-0.6 \%, p=0.03)$ but not the low-fat diet group $(-0.5 \%, \mathrm{p}=0.10)$.

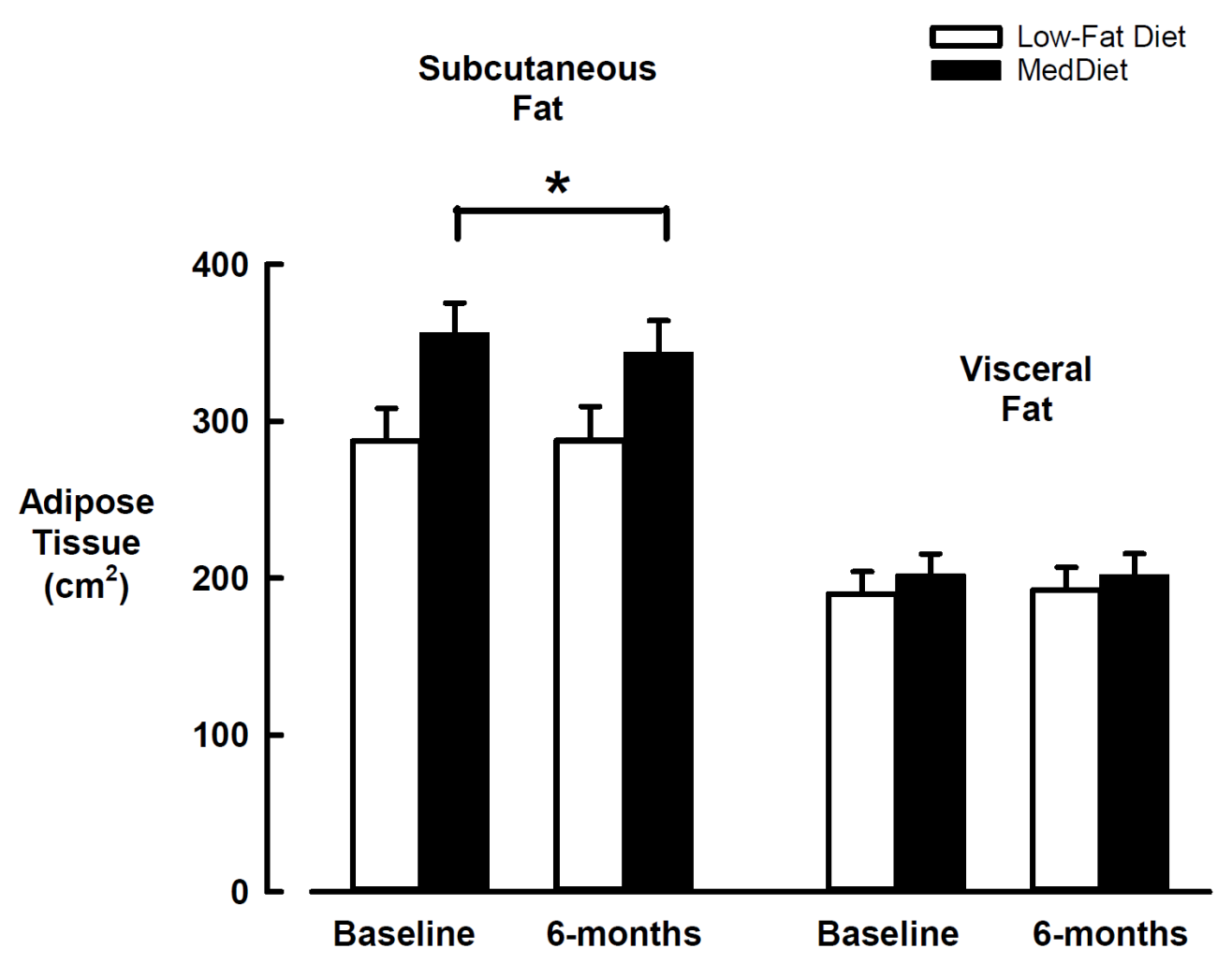

317 Figure 1. Subcutaneous and visceral adipose tissue measured by dual energy $x$-ray absorptiometry at 318 baseline and end-intervention in the MedDiet and low-fat diet groups. Data are mean \pm SEM with 319 adjustment for change in moderate to vigorous physical activity levels. MedDiet, Mediterranean diet. $320 *$ Significant reduction in MedDiet compared to low-fat diet participants, $p=0.04$.

\subsection{Haemodynamic, Cholesterol and Glucose Measures}

323 There were no between-group changes for any of the reported haemodynamic, cholesterol or glucose markers, 324 adjusted for MVPA change and baseline BMI (Table 2). There was a significant reduction in resting HR in the 325 pooled study groups $(p=0.03)$, with a trend for greater reduction in the MedDiet $(-1.5 \mathrm{bpm}, \mathrm{p}=0.07)$ compared 326 to the low-fat diet $(-0.8 \mathrm{bpm}, \mathrm{p}=0.55)$ group. With regards to lipids, the only significant within-group finding 327 was an increase in LDL cholesterol between baseline and 6-months $(+0.22 \mathrm{mmol} / \mathrm{L}, p=0.006)$ in the low-fat 
diet group. There were no changes within either study group for triglycerides or fasting glucose levels (also assessed separately for T2DM status).

\subsection{Adiponectin}

332 There were no between-group changes for serum levels of the anti-inflammatory marker adiponectin $(p=0.45)$

333 adjusted for MVPA change and baseline BMI (Table 2). There was also no significant change in adiponectin 334 between baseline and 6-months within the low-fat diet $(-0.91 \mathrm{ng} / \mathrm{mL}, p=0.23)$ or MedDiet $(+1.10 \mathrm{ng} / \mathrm{mL}$, $335 p=0.37$ ) groups. Data from this interim analysis on the 6-month between-within group changes for adiponectin were used to perform a reverse sample size calculation in statistical software program $G^{*}$ Power 3.1.94 (49).

337 Based on the study group effect size (derived from the partial eta ${ }^{2}$ of the repeated measures group comparison) 338 of 0.101 , and a correlation value between adiponectin levels at baseline and 6-months of $\mathrm{r}=0.689$ at $80 \%$ power 339 and $\alpha<0.05$, a sample size of 124 participants would be required to detect a significant effect of the MedDiet 340 on adiponectin compared to the low-fat diet.

\subsection{Malondialdehyde}

343 There were no between-group changes for plasma MDA levels $(p=0.75)$ adjusted for MVPA change and 344 baseline BMI (Table 2). There was also no significant change in MDA levels between baseline and 6-months 345 within the low-fat diet $(+0.02 \mathrm{nmol} / \mathrm{mL}, p=0.93)$ or MedDiet $(-0.25 \mathrm{nmol} / \mathrm{L}, p=0.24)$ groups. MDA data were 346 also used to perform a sample size calculation as above. Based on the study group effect size of 0.045 and a correlation value between MDA levels at baseline and 6-months of $r=0.775$, a sample size of 444 participants would be required to detect a significant effect of the MedDiet on MDA levels compared to the low-fat diet. 
Table 2. Cardiometabolic risk markers at baseline and end-intervention in the study groups

\begin{tabular}{|c|c|c|c|c|c|c|}
\hline \multirow[t]{2}{*}{ Marker } & \multicolumn{2}{|c|}{ Low-fat diet $(n=31)$} & \multicolumn{2}{|c|}{ MedDiet $(n=34)$} & \multicolumn{2}{|l|}{$p$-value } \\
\hline & Baseline & 6-month & Baseline & 6-month & Group & Time \\
\hline \multicolumn{7}{|l|}{ Activity levels } \\
\hline Sedentary (min/week) & $3559 \pm 135$ & $3431 \pm 138$ & $3477 \pm 133$ & $3380 \pm 135$ & 0.70 & 0.18 \\
\hline MVPA (min/week) & $148 \pm 27$ & $135 \pm 21$ & $186 \pm 26$ & $144 \pm 20$ & 0.33 & 0.37 \\
\hline \multicolumn{7}{|l|}{ Anthropometry } \\
\hline Weight (kg) & $85.0 \pm 3.3$ & $84.7 \pm 3.3$ & $89.1 \pm 3.2$ & $89.1 \pm 3.1$ & 0.36 & 0.51 \\
\hline Body mass index $\left(\mathrm{kg} / \mathrm{m}^{2}\right)$ & $29.0 \pm 0.9$ & $28.9 \pm 0.9$ & $30.8 \pm 0.9$ & $30.8 \pm 0.9$ & 0.15 & 0.58 \\
\hline Waist circumference $(\mathrm{cm})$ & $101.9 \pm 2.6$ & $101.5 \pm 2.5$ & $104.5 \pm 2.5$ & $103.4 \pm 2.4$ & 0.53 & 0.07 \\
\hline Waist-hip ratio & $0.976 \pm 0.02$ & $0.973 \pm 0.01$ & $0.977 \pm 0.01$ & $0.966 \pm 0.01$ & 0.89 & 0.10 \\
\hline \multicolumn{7}{|l|}{ Body composition } \\
\hline Total lean (kg) & $55.6 \pm 2.0$ & $55.9 \pm 2.0$ & $56.1 \pm 1.0$ & $56.6 \pm 1.9$ & 0.83 & 0.22 \\
\hline Total fat (kg) & $27.7 \pm 1.8$ & $27.2 \pm 1.7$ & $31.1 \pm 1.7$ & $30.6 \pm 1.6$ & 0.16 & 0.11 \\
\hline Fat mass index $\left(\mathrm{kg} / \mathrm{m}^{2}\right)$ & $9.45 \pm 0.6$ & $9.42 \pm 0.6$ & $10.84 \pm 0.5$ & $10.68 \pm 0.6$ & 0.10 & 0.42 \\
\hline Total fat $\%$ & $32.7 \pm 1.2$ & $32.3 \pm 1.3$ & $35.3 \pm 1.1$ & $34.7 \pm 1.2$ & 0.13 & 0.05 \\
\hline Trunk fat $\%$ & $34.9 \pm 1.2$ & $34.6 \pm 1.3$ & $37.9 \pm 1.1$ & $37.3 \pm 1.2$ & 0.10 & 0.08 \\
\hline Arm fat $\%$ & $32.7 \pm 1.6$ & $32.2 \pm 1.7$ & $36.2 \pm 1.5$ & $35.8 \pm 1.6$ & 0.12 & 0.21 \\
\hline Leg fat $\%$ & $30.6 \pm 1.4$ & $30.1 \pm 1.5$ & $32.6 \pm 1.3$ & $32.0 \pm 1.4^{\mathrm{a}}$ & 0.31 & $0.04 *$ \\
\hline \multicolumn{7}{|l|}{ Haemodynamic } \\
\hline $\mathrm{SBP}(\mathrm{mmHg})$ & $140.6 \pm 3.3$ & $139.5 \pm 2.8$ & $133.4 \pm 3.1$ & $132.0 \pm 2.6$ & 0.07 & 0.84 \\
\hline DBP (mmHg) & $83.3 \pm 1.6$ & $83.0 \pm 1.6$ & $81.0 \pm 1.5$ & $80.6 \pm 1.5$ & 0.25 & 0.09 \\
\hline HR (bpm) & $66.9 \pm 2.0$ & $66.1 \pm 2.0$ & $68.5 \pm 1.9$ & $66.0 \pm 1.9$ & 0.77 & $0.03 *$ \\
\hline \multicolumn{7}{|l|}{ Pathology } \\
\hline $\mathrm{LDL}(\mathrm{mmol} / \mathrm{L}) \dagger$ & $1.73 \pm 0.13$ & $1.95 \pm 0.15^{\mathrm{a}}$ & $1.96 \pm 0.13$ & $1.99 \pm 0.15$ & 0.48 & 0.35 \\
\hline $\mathrm{HDL}(\mathrm{mmol} / \mathrm{L}) \dagger$ & $1.20 \pm 0.05$ & $1.24 \pm 0.05$ & $1.21 \pm 0.05$ & $1.25 \pm 0.05$ & 0.83 & 0.47 \\
\hline Triglycerides $(\mathrm{mmol} / \mathrm{L}) \dagger$ & $1.35 \pm 0.13$ & $1.30 \pm 0.14$ & $1.57 \pm 0.12$ & $1.60 \pm 0.13$ & 0.12 & 0.65 \\
\hline Glucose (mmol/L) & $5.27 \pm 0.26$ & $5.18 \pm 0.27$ & $5.76 \pm 0.25$ & $5.65 \pm 0.26$ & 0.17 & 0.13 \\
\hline No T2DM $(n=44)$ & $4.92 \pm 0.16$ & $4.78 \pm 0.12$ & $4.98 \pm 0.15$ & $4.91 \pm 0.11$ & 0.60 & 0.17 \\
\hline T2DM $(n=19) \ddagger$ & $6.20 \pm 0.47$ & $6.11 \pm 0.67$ & $7.49 \pm 0.45$ & $7.38 \pm 0.63$ & 0.08 & 0.31 \\
\hline
\end{tabular}




$\begin{array}{lllllll}\text { Adiponectin }(\mathrm{ng} / \mathrm{mL}) \dagger & 8.40 \pm 0.71 & 7.49 \pm 0.83 & 8.36 \pm 0.68 & 9.47 \pm 0.81 & 0.45 & 0.65 \\ \text { Malondialdehyde }(\mathrm{nmol} / \mathrm{mL}) & 6.96 \pm 0.31 & 6.98 \pm 0.33 & 6.96 \pm 0.30 & 6.71 \pm 0.32 & 0.75 & 0.11\end{array}$

Values are Mean \pm SEM with anthropometry/body composition adjusted for MVPA change, and

358 haemodynamic/pathology markers adjusted for MVPA change and baseline BMI. One low-fat diet participant did not consent to DXA scan and was excluded from body composition analyses. One MedDiet participant who dropped out and had haemolysed blood sample at baseline was excluded from pathology marker analyses. MedDiet, Mediterranean diet; MVPA; moderate to vigorous physical activity; SBP; systolic blood pressure; DBP, diastolic blood pressure; HR, heart rate; LDL, low-density lipoprotein; HDL, high-density lipoprotein; T2DM, type 2 diabetes mellitus; †Non-parametric, analyses based on transformed variable. $†$ One participant with T2DM had a major increase in insulin dosage and was excluded from analyses. Significant, $p<0.05$, for: *Main effect of group or time;

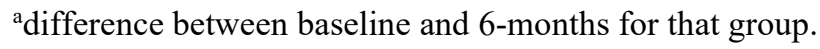

\subsection{Association Between Mediterranean Diet Adherence and Risk Markers}

368 All participants were categorised into tertiles of change in MEDAS score from baseline to 6-months. This resulted in tertile 1 (T1) of -2 to +1 , tertile 2 (T2) of +2 to 5 , and tertile 3 (T3) of +6 to 9 . As expected, in T3, with the largest 6-month improvement in MedDiet adherence, 93\% of participants were from the MedDiet group. In T2 and T1 the proportion of participants in the MedDiet group was 56\% and 22\%, respectively. Mean (95\% CI) levels for cardiometabolic risk markers at 6-months, adjusted for baseline value, sex, age, T2DM, 373 time since coronary event and change in MVPA, are presented in Table 3. For each of the reported 374 anthropometric, body composition and hemodynamic measures the mean value decreased across tertiles from T1 to T3 (from lowest to greatest MEDAS score improvement), except for VAT area and DBP, which had a higher mean value in T2, followed by T1 and then T3. Compared to T1, T3 participants had a significantly lower mean waist circumference $(-2.81 \mathrm{~cm}, p=0.01)$, waist-hip ratio $(-0.022, \mathrm{p}=0.047)$ and SAT area $(-27.4$ $\left.378 \mathrm{~cm}^{2}, \mathrm{p}=0.04\right)$. Mean levels of other pathology markers did not demonstrate any consistent trends across tertiles. 379 For adiponectin, the mean value increased slightly but with no significant difference across tertiles from lowest 380 to greatest MEDAS score improvement $(+0.68 \mathrm{ng} / \mathrm{mL}$ from $\mathrm{T} 1$ to $\mathrm{T} 3, p=0.50)$. There was also no difference 381 between tertiles for plasma MDA level $(-0.31 \mathrm{nmol} / \mathrm{mL}$ from $\mathrm{T} 1$ to $\mathrm{T} 3, \mathrm{p}=0.46)$. 
383 Table 3. Adjusted means of cardiometabolic risk markers at 6-months by tertiles of change in 384 Mediterranean Diet Adherence Screener (MEDAS) score ${ }^{\mathrm{a}}$

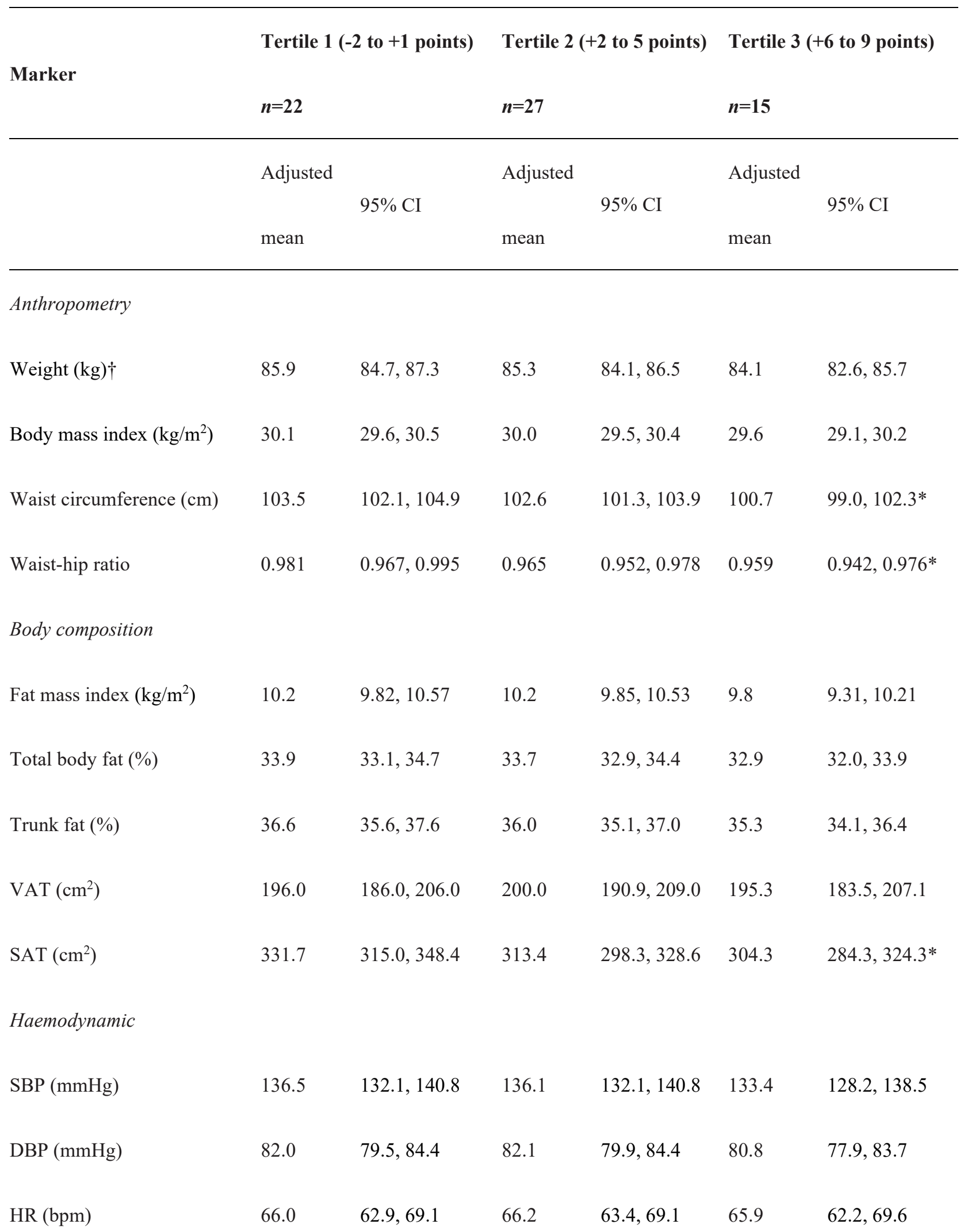




\section{Pathology $\dagger$}

$\begin{array}{lcccccc}\text { LDL (mmol/L) } & 1.69 & 1.51,1.90 & 1.97 & 1.77,2.17 & 1.70 & 1.49,1.95 \\ \text { HDL (mmol/L) } & 1.26 & 1.19,1.33 & 1.19 & 1.14,1.25 & 1.19 & 1.11,1.27 \\ \text { Triglycerides (mmol/L) } & 1.16 & 1.01,1.33 & 1.37 & 1.22,1.55 & 1.30 & 1.11,1.53 \\ \text { Glucose (mmol/L)* } & 5.09 & 4.79,5.41 & 5.32 & 5.06,5.61 & 5.50 & 5.12,5.87 \\ \text { hs-CRP (mg/L)** } & 0.69 & 0.43,1.09 & 0.83 & 0.54,1.27 & 0.87 & 0.50,1.50 \\ & 1.42 & 1.09,1.99 & 1.46 & 1.09,1.96 & 1.46 & 0.99,2.15 \\ \text { hs-IL-6 (pg/mL) } & & & & & & \\ \text { Adiponectin (ng/mL) } & 7.19 & 6.08,8.51 & 7.62 & 6.44,9.59 & 7.87 & 6.44,9.59 \\ \text { Malondialdehyde (nmol/mL) } & 6.77 & 6.24,7.30 & 7.11 & 6.63,7.58 & 6.46 & 5.84,7.08\end{array}$

385 T, tertile; CI; confidence interval; VAT visceral adipose tissue; SAT, subcutaneous adipose tissue; SBP, systolic blood

386 pressure; DBP, diastolic blood pressure; HR, heart rate; LDL, low-density lipoprotein; HDL, high-density lipoprotein;

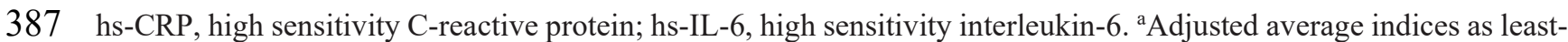

388 square means with 95\%CI adjusted for baseline value, sex, age, type 2 diabetes mellitus, time since coronary event

389 and change in moderate-to-vigorous activity levels. *Significant difference between T1 and T3, $p<0.05$. † Variable

390 log-transformed and data are presented as adjusted geometric means and confidence intervals have been backwards

391 logged, except for MDA. †One participant with T2DM had a major increase in insulin dosage and was excluded from

392 analyses. **Two participants excluded for value $>10 \mathrm{mg} / \mathrm{L}$.

\section{Discussion}

397 This study has reported on the effect of a 6-month intervention with ad libitum MedDiet versus low-fat diet on 398 adiposity, anti-inflammatory marker adiponectin and oxidative stress marker MDA in a cohort of patients with 
CHD. The results demonstrated that the MedDiet significantly reduced SAT but not VAT area compared to 400 the low-fat diet. Despite significantly improved adherence to the Mediterranean dietary pattern (29), there was 401 no significant effect of the MedDiet on adiponectin, MDA or classic CVD risk markers of lipids, glucose or 402 blood pressure compared with the low-fat diet. Notable within group findings were a reduction in the 403 proportion of MedDiet participants prescribed $\beta$-blockers and increased LDL cholesterol levels in the low-fat 404 diet group. Across tertiles of increasing improvement in MedDiet adherence score in the pooled study cohort at 6-months, a significantly lower waist circumference, WHR and SAT area was observed.

407 The significant improvement in the SAT but not the VAT area following MedDiet compared to the low-fat 408 diet was unexpected. Two previous studies $(50,51)$ reported a significant reduction in markers of VAT 409 (measured by bioelectrical impedance analysis or ultrasound) following MedDiet intervention. One of these 410 studies also demonstrated that MedDiet intervention did not significantly impact subcutaneous fat (50). Both 411 previous interventions employed energy restrictions and were conducted in patients without CVD, which may 412 explain why no reduction in VAT was observed in the current study of an ad libitum MedDiet and the first in 413 CHD patients. The reduction in subcutaneous fat in the present study is contradictory to previous findings that 414 intake of MUFA (which AUSMED participants substantially increased) favours deposition as subcutaneous 415 rather than visceral fat (52). The lack of improvement in VAT with our MedDiet intervention assists to explain 416 the lack of significant effect on inflammatory markers given VAT represents more metabolically dysfunctional 417 tissue $(16,17)$. There is an established protective effect of exercise on visceral fat (53) and chronic 418 inflammation in patients with CHD (54). Therefore, whilst changes in MVPA were controlled for, it cannot be 419 ruled out that a lack of improvement in VAT area and inflammatory biomarkers was related to an observed 420 reduction in MVPA levels in some MedDiet participants.

422 The maintenance of weight and trend for reduction in total body fat in the MedDiet group occurred despite the 423 tendency of the group to increase total energy intake (29). These findings assist to discount the belief that the 424 high healthy fat MedDiet is associated with weight and fat gain (55) and could be related to the high content of unsaturated fats, particularly MUFA and omega-3 PUFA, in the MedDiet. These unsaturated fats have been 426 shown to be associated with increased lipid oxidation and thermic effect $(56,57)$. Furthermore, in a cohort of 
Australian patients with T2DM ( $n=27)$ a 12 -week ad libitum MedDiet intervention resulted in a small reduction

428 in body weight, despite significantly increased energy and MUFA intake (13).

430 This is the first study to examine the effect of MedDiet on the anti-inflammatory marker adiponectin in patients 431 with diagnosed CHD. No significant change was detected in this pilot cohort, with only a trend for reduction 432 in the MedDiet compared to low-fat diet group observed. Adiponectin has been reported in previous MedDiet 433 intervention studies that have been conducted in subject groups without CHD diagnosis. In a study of pre434 menopausal obese women adiponectin increased with a calorie-restricted MedDiet compared to general 435 diet/exercise advice (58). A sub-study of the PREDIMED trial in patients with T2DM also demonstrated an 436 increase in plasma adiponectin, but this increase occurred with all three (Mediterranean + EVOO, 437 Mediterranean + Nuts and low-fat) diet interventions; mean weight loss was significant but less than $1 \mathrm{~kg}$ in 438 each group (9). It was also found that a MedDiet in the absence of weight loss can significantly reduce 439 inflammation (composite score of CRP, IL-6 and tumour necrosis factor- $\alpha$ ) (59) but not levels of adiponectin 440 (60). The DIRECT study, which included a MedDiet intervention with 6-month weight loss phase followed by 441 an 18-month weight maintenance phase, demonstrated a continued significant increase in adiponectin for the 442 duration of the trial (61). Most of these findings suggest that a significant increase in adiponectin with MedDiet 443 is dependent on concomitant weight loss (at least initially), which helps to explain the lack of significant effect 444 on adiponectin in the current study with an ad libitum approach. Our results estimated that without weight loss 445 (and no change in VAT), twice the sample size would be required to demonstrate a significant improvement 446 in adiponectin with MedDiet compared to low-fat diet.

448 This was one of first studies to examine the effect of a dietary intervention on oxidative stress marker plasma 449 MDA in patients with CHD and no significant effect of the MedDiet compared to low-fat diet was found. 450 Similarly, a previous study of MedDiet intervention versus control (habitual) diet in patients with Rheumatoid 451 Arthritis and on stable pharmacological treatment $(n=51)$ demonstrated no effect on MDA levels in urine (62). 452 A preliminary study in a subset of PREDIMED participants $(n=71)$, at high risk of but without CHD, did 453 demonstrate a significant improvement in MDA with 3-month MedDiet versus low-fat diet (27). However, 454 MDA was measured from peripheral blood mononuclear cells (rather than circulating levels as measured in 
our study) and the changes in MDA paralleled improvements in oxidised LDL levels. Recent meta-analysis

456 also demonstrated evidence to support that consumption of extra virgin olive oil reduces MDA levels, however, 457 the four intervention studies each prescribed $\sim 70 \mathrm{~g}$ oil per day and were conducted in healthy adults (63).

Of particular interest, a significant number of MedDiet participants (15\%) stopped taking $\beta$-blocker medication during the trial. Despite cessation of this antihypertensive medication, for the MedDiet cohort no significant change in mean SBP, DBP or HR was detected. A potential reduction in need for this medication with the MedDiet is a promising finding as $\beta$-blockers have a range of short and long-term side effects (44). This finding warrants for the effect of the MedDiet on cardiac medication to be investigated further. The present study also 464 demonstrated no significant effect of the MedDiet on LDL cholesterol, triglycerides or glucose, compared to 465 the low-fat diet. These results were not unexpected considering that most participants were prescribed statins 466 or other lipid-lowering therapy as well as anti-hypertensives, and nearly all participants with T2DM were 467 taking hypoglycaemic agents. Interestingly, the low-fat diet group significantly increased LDL cholesterol levels after 6-months. This contradicts the premise of the low-fat diet, which was designed to lower LDL 469 cholesterol levels. This finding may be reflective of the lack of improvement in adherence to the low-fat diet principles seen in that group, and their slight increase in saturated fat intake (29).

Our study had a number of strengths. The intensity of the dietary counselling was the same in both groups to control for this effect. In both study groups the focus of the intervention was dietary improvement only and the approach was ad libitum in order to isolate the effect of diet rather than changes in weight loss or improved physical activity. The secondary analyses in the pooled cohort allowed for the potential effect of greater magnitude of improvement in adherence to the MedDiet pattern, including within the low-fat diet group, to be explored. We also demonstrated that there were no significant differences in access to other health services or 478 changes in types of medication or supplements taken between the groups, except for a reduction in use of $\beta$ 479 blockers in the MedDiet group. Finally, intention-to-treat analyses were performed which meant that dropouts were accounted for in all analyses. 
This study was however limited by the small size of a preliminary cohort of AUSMED participants, and hence was underpowered. Based on the results in these patients, the reverse power calculations which were performed for novel markers adiponectin and MDA estimated that a sample size double and close to seven times that of the current sample would be required to detect a significant effect of the ad libitum MedDiet compared to lowfat diet on these markers respectively in a CHD patient setting. These results will inform future studies and analyses. The patients recruited in this study represent a lower proportion of females and are potentially more health conscious/motivated than ACS patients in the broader Australian population (64), which may impact the generalisability of the results. Nonetheless, the results of this study may be generalisable to other nonMediterranean, multicultural populations. Finally, whilst Hologic DXA measurement for VAT and SAT area are validated, they provide an estimate only.

\section{Conclusions}

In a small cohort of Australian patients with CHD a 6-month ad libitum MedDiet nor low-fat diet intervention led to significant improvement in adiponectin or plasma MDA levels. A lack of significant change in weight and trends for improved body fat and waist circumference assists to discount the continued misconception that a diet high in healthy fats, such is the MedDiet, leads to weight or fat gain. Greater improvement in MedDiet adherence was associated with lower waist circumference, however, this was associated with lower SAT and not VAT area, which was unexpected and may explain a lack of significant effect on measured cardiometabolic risk markers. Future studies are needed in larger cohorts. Nonetheless, in CHD patients taking intensive medications, significant clinical effects of the MedDiet on these markers may require adjunct exercise intervention and/or caloric restriction.

\section{Declarations}

\section{Ethics approval and consent to participate}

This study was approved by the Human Research Ethics Committees of the Northern Hospital (HREC/16/Austin/500), St Vincent's Hospital Melbourne (HREC-A; 016/13), and La Trobe University (\#FHEC13/159). Written informed consent was obtained from all participants. 
Availability of data and materials

512 The datasets used and/or analysed during the current study are available from the corresponding author on 513 reasonable request.

Competing interests

The Mediterranean Diet by Itsiopoulos (2013) (ISBN 9781742610825) was provided to the Mediterranean diet group participants as a dietary resource. Otherwise the authors have no conflicts of interest to declare.

\section{$519 \quad$ Funding}

520 This research was funded by La Trobe University Understanding Disease RFA Start-Up Grant, 2013 and La 521 Trobe University Deputy Vice-Chancellor (Research) Near-Miss NHMRC Grant, 2018. HLM was supported by an Australian Government Research Training Program Scholarship and a Northern Health PhD Scholarship. The Funding bodies had no role in the design of the study; collection, analysis, and interpretation of data; or in writing the manuscript.

Authors' contributions

TK, CI and ACT conceptualised and designed the research. HLM collected the presented data, analysed data and interpreted results (with support from, CI, ACT, JR, CJT, MG and JW). HLM, JR and MG were involved in laboratory analyses of pathology markers. HLM wrote the draft manuscript. All authors critically reviewed, edited and approved the manuscript.

Acknowledgements

533 The roles of the sponsors are as follows: the supplemental foods used in the study were generously donated by 534 Cobram Estate Pty Ltd (extra virgin olive oil); the Almond Board of Australia (almonds); Jalna Dairy Foods 535 Pty Ltd (Greek yoghourt); Simplot Australia Pty Ltd (canned fish and legumes); HJ Heinz Company Australia 
537 in the collection, analyses, or interpretation of data; in the writing of the manuscript, and in the decision to 538 publish the results.

540 The authors are very grateful to all the participants of the study for their enthusiastic involvement and to the 541 personnel of the affiliated hospital sites. We thank Dr. Elena George for her work in designing the 542 Mediterranean diet and low-fat diet interventions of this study (alongside co-author TK); Ms. Cassandra 543 Bendall for her assistance with data collection and entry, and laboratory analyses for adiponectin; Diana 544 Navarro-Perez for assistance with laboratory analyses of pathology markers; and Mrs. Elizabeth Kennedy for 545 her assistance with recruitment of participants and conducting appointments

547 Supplementary Materials

548 CONSORT 2010 checklist of information to include when reporting a randomised trial. Completed for current 549 AUSMED study.

550 Table S1. Proportion of participants taking prescribed medications and supplements across intervention time 551 points in the study groups.

552 Table S2. Frequency of attendance for study appointments and phone reviews and access to other health 553 services during the intervention period in the total cohort and within study groups. 
555 1. de Lorgeril M, Salen P, Martin J-L, Monjaud I, Delaye J, Mamelle N. Mediterranean diet, 556 traditional risk factors, and the rate of cardiovascular complications after myocardial infarction. 557 Circulation. 1999;99(6):779-85.

558 2. Estruch R, Ros E, Salas-Salvadó J, Covas M-I, Corella D, Arós F, et al. Primary prevention of 559 cardiovascular disease with a mediterranean diet supplemented with extra-virgin olive oil or nuts. $\mathrm{N}$ $560 \quad$ Engl J Med. 2018;378(25):e34.

561 3. National Heart Foundation of Australia. Reducing risk in heart disease: an expert guide to clinical 562 practice for secondary prevention of coronary heart disease. Melbourne: National Heart Foundation 563 of Australia and the Cardiac Society of Australia and New Zealand; 2012.

564 4. Natrional Heart Foundation of Australia. Eating for Heart Health Position Statement. Melbourne: 565 National Heart Foundation of Australia; 2017.

5665 5inlay S, Libby P, Ganz P. Endothelial function and coronary artery disease. Curr Opin Lipidol. $567 \quad 2001 ; 12(4): 383-9$.

568 6. Hernaez A, Castaner O, Elosua R, Pinto X, Estruch R, Salas-Salvado J, et al. Mediterranean Diet 569 Improves High-Density Lipoprotein Function in High-Cardiovascular-Risk Individuals: A 570 Randomized Controlled Trial. Circulation. 2017;135(7):633-43.

571 7. Davis CR, Bryan J, Hodgson JM, Woodman R, Murphy KJ. A Mediterranean Diet Reduces F2572 Isoprostanes and Triglycerides among Older Australian Men and Women after 6 Months. J Nutr. $573 \quad 2017 ; 147(7): 1348-55$. 
8. Estruch R, Martínez-González MAn, Corella D, Salas-Salvadó J, Ruiz-Gutiérrez V, Covas MaI, et al. Effects of a Mediterranean-Style Diet on Cardiovascular Risk Factors: A Randomized Trial. Ann Intern Med. 2006;145(1):1-11.

9. Lasa A, Miranda J, Bullo M, Casas R, Salas-Salvado J, Larretxi I, et al. Comparative effect of two Mediterranean diets versus a low-fat diet on glycaemic control in individuals with type 2 diabetes. Eur J Clin Nutr. 2014;68(7):767-72.

10. Vincent-Baudry S, Defoort C, Gerber M, Bernard M-C, Verger P, Helal O, et al. The MediRIVAGE study: reduction of cardiovascular disease risk factors after a 3-mo intervention with a Mediterranean-type diet or a low-fat diet. Am J Clin Nutr. 2005;82(5):964-71.

11. Salas-Salvadó J, Bulló M, Babio N, Martínez-González MÁ, Ibarrola-Jurado N, Basora J, et al. Reduction in the incidence of type 2 diabetes with the Mediterranean diet. Diabetes Care. 2011;34(1):14-9.

12. Davis CR, Hodgson JM, Woodman R, Bryan J, Wilson C, Murphy KJ. A Mediterranean diet lowers blood pressure and improves endothelial function: results from the MedLey randomized intervention trial. Am J Clin Nutr. 2017;105(6):1305-13.

13. Itsiopoulos C, Brazionis L, Kaimakamis M, Cameron M, Best JD, O’Dea K, et al. Can the Mediterranean diet lower HbA1c in type 2 diabetes? Results from a randomized cross-over study. Nutr Metab Cardiovasc Dis. 2011;21(9):740-7.

14. Chew DP, Scott IA, Cullen L, French JK, Briffa TG, Tideman PA, et al. National Heart Foundation of Australia and Cardiac Society of Australia and New Zealand: Australian clinical guidelines for the management of acute coronary syndromes 2016. Med J Aust. 2016;205(3):128-33. 
15. Ross R. Atherosclerosis — An Inflammatory Disease. N Engl J Med. 1999;340(2):115-26.

16. Nakamura K, Fuster JJ, Walsh K. Adipokines: a link between obesity and cardiovascular disease. J Cardiol. 2014;63(4):250-9.

17. Samaras K, Botelho NK, Chisholm DJ, Lord RV. Subcutaneous and visceral adipose tissue gene expression of serum adipokines that predict type 2 diabetes. Obesity. 2010;18(5):884-9. 18. Ouchi N, Parker JL, Lugus JJ, Walsh K. Adipokines in inflammation and metabolic disease. Nat $601 \quad$ Rev Immunol. 2011;11(2):85-97. 19. Schulze MB, Shai I, Rimm EB, Li T, Rifai N, Hu FB. Adiponectin and future coronary heart 603 disease events among men with type 2 diabetes. Diabetes. 2005;54(2):534-9.

604 20. Pischon T, Girman CJ, Hotamisligil GS, Rifai N, Hu FB, Rimm EB. Plasma adiponectin levels 605 and risk of myocardial infarction in men. JAMA. 2004;291(14):1730-7.

606 21. Heitzer T, Schlinzig T, Krohn K, Meinertz T, Münzel T. Endothelial dysfunction, oxidative 607 stress, and risk of cardiovascular events in patients with coronary artery disease. Circulation. $608 \quad 2001 ; 104(22): 2673-8$.

609 22. Mutlu-Türkoðlu Ü, Akalýn Z, Ýlhan E, Yýlmaz E, Bilge A, Nipancý Y, et al. Increased plasma 610 malondialdehyde and protein carbonyl levels and lymphocyte DNA damage in patients with 611 angiographically defined coronary artery disease. Clin Biochem. 2005;38(12):1059-65.

612 23. Bendall CL, Mayr HL, Opie RS, Bes-Rastrollo M, Itsiopoulos C, Thomas CJ. Central obesity 613 and the Mediterranean diet: A systematic review of intervention trials. Crit Rev Food Sci Nutr. $614 \quad 2017: 1-15$. 
615 24. Neale E, Batterham M, Tapsell LC. Consumption of a healthy dietary pattern results in significant 616 reductions in C-reactive protein levels in adults: a meta-analysis. Nutr Res. 2016;36(5):391-401.

617 25. Schwingshackl L, Hoffmann G. Mediterranean dietary pattern, inflammation and endothelial 618 function: a systematic review and meta-analysis of intervention trials. Nutr Metab Cardiovasc Dis. 619 2014;24(9):929-39.

620 26. Mayr HL, Tierney AC, Thomas CJ, Ruiz-Canela M, Radcliffe J, Itsiopoulos C. Mediterranean621 type diets and inflammatory markers in patients with coronary heart disease: a systematic review and 622 meta-analysis. Nutr Res. 2018;50(Supplement C):10-24.

623 27. Fito M, Guxens M, Corella D, Saez G, Estruch R, de la Torre R, et al. Effect of a traditional 624 Mediterranean diet on lipoprotein oxidation: a randomized controlled trial. Arch Intern Med. $625 \quad 2007 ; 167(11): 1195-203$.

626 28. Mayr HL, Thomas CJ, Tierney AC, Kucianski T, George ES, Ruiz-Canela M, et al. 627 Randomization to 6-month Mediterranean diet compared with a low-fat diet leads to improvement in 628 Dietary Inflammatory Index scores in patients with coronary heart disease: the AUSMED Heart Trial. 629 Nutr Res. 2018;55:94-107.

630 29. Mayr HL, Tierney AC, Kucianski T, Thomas CJ, Itsiopoulos C. Australian patients with coronary 631 heart disease achieve high adherence to 6-month Mediterranean diet intervention: preliminary results 632 of the AUSMED Heart Trial. Nutrition. 2019;16(2019):21-31.

633 30. Itsiopoulos C, Kucianski T, Mayr HL, van Gaal WJ, Martinez-Gonzalez MA, Vally H, et al. The 634 AUStralian MEDiterranean diet heart trial (AUSMED heart trial): A randomized clinical trial in 

secondary prevention of coronary heart disease in a multi-ethnic Australian population: Study 636 protocol. Am Heart J. 2018;203(2018):4-11.

637 31. Mayr HL, Itsiopoulos C, Tierney AC, Ruiz-Canela M, Hebert JR, Shivappa N, et al. 638 Improvement in dietary inflammatory index score after 6-month dietary intervention is associated 639 with reduction in interleukin-6 in patients with coronary heart disease: The AUSMED heart trial. Nutr $640 \quad$ Res. 2018;55:108-21.

641 32. World Medical Association. World Medical Association Declaration of Helsinki: ethical 642 principles for medical research involving human subjects. Available online: 643 https://www.wma.net/what-we-do/medical-ethics/declaration-of-helsinki/ $\quad$ (accessed on 14 644 February 2018) 2008 [

645 33. Schulz KF, Altman DG, Moher D. CONSORT 2010 statement: updated guidelines for reporting 646 parallel group randomised trials. BMC Med. 2010;8(1):18.

647 34. Papamiltiadous ES, Roberts SK, Nicoll AJ, Ryan MC, Itsiopoulos C, Salim A, et al. A 648 randomised controlled trial of a Mediterranean Dietary Intervention for Adults with Non Alcoholic 649 Fatty Liver Disease (MEDINA): study protocol. BMC Gastroenterol. 2016;16(1):14.

650 35. George ES, Kucianski T, Mayr HL, Moschonis G, Tierney AC, Itsiopoulos C. A Mediterranean 651 Diet Model in Australia; Strategies for Translating the Traditional Mediterranean Diet into a 652 Multicultural Setting. Nutrients. 2018;10(4):465.

653 36. National Health and Medical Research Council. Australian Dietary Guidelines. Canberra: 654 National Health and Medical Research Council; 2013. 
37. National Health and Medical Research Council. Nutrient Reference Values for Australia and 656 New Zealand; Including Recommended Dietary Intakes. Canberra: Commenwealth of Australia; 6572006.

658 38. Schröder H, Fitó M, Estruch R, Martínez-González MA, Corella D, Salas-Salvadó J, et al. A 659 Short Screener Is Valid for Assessing Mediterranean Diet Adherence among Older Spanish Men and 660 Women. J Nutr. 2011;141(6):1140-5.

661 39. Freedson PS, Melanson E, Sirard J. Calibration of the Computer Science and Applications, Inc. 662 accelerometer. Med Sci Sports Exerc. 1998;30(5):777-81.

663 40. ISAK. International Standards for Anthropometric Assessment. South Australia: International 664 Society for the Advancement of Kinanthropometry; 2001.

665 41. Kelly T, Wilson KE, Ruth C. Estimating visceral fat by dual-energy X-ray absorptiometry. 666 Google Patents; 2015.

667 42. Micklesfield LK, Goedecke JH, Punyanitya M, Wilson KE, Kelly TL. Dual-Energy X-Ray 668 performs as well as clinical computed tomography for the measurement of visceral fat. Obesity. $6692012 ; 20(5): 1109-14$.

670 43. Kelly TL, Wilson KE, Heymsfield SB. Dual energy X-Ray absorptiometry body composition 671 reference values from NHANES. PLoS One. 2009;4(9):e7038.

672 44. National Heart Foundation of Australia. Guideline for the diagnosis and management of 673 hypertension in adults — 2016. Melbourne: National Heart Foundation of Australia; 2016.

674 45. Mayr HL. The Effect of a Mediterranean Diet Versus Low-fat Diet on Inflammation and 675 Adiposity: an Intermediate Analysis of the AUSMED Heart Trial for Secondary Prevention of 
677 June 2018.

678 46. Eldridge SM, Chan CL, Campbell MJ, Bond CM, Hopewell S, Thabane L, et al. CONSORT 6792010 statement: extension to randomised pilot and feasibility trials. Pilot and feasibility studies. $680 \quad 2016 ; 2(1): 64$.

681 47. Liu-Seifert H, Zhang S, D'Souza D, Skljarevski V. A closer look at the baseline-observation682 carriedforward (BOCF). Patient Prefer Adherence. 2010;4:11-6.

683 48. Pearson TA, Mensah GA, Alexander RW, Anderson JL, Cannon RO, Criqui M, et al. Markers 684 of Inflammation and Cardiovascular Disease. Application to Clinical and Public Health Practice: A 685 Statement for Healthcare Professionals From the Centers for Disease Control and Prevention and the 686 American Heart Association. 2003;107(3):499-511.

49. Faul F, Erdfelder E, Lang A-G, Buchner A. G*Power 3: A flexible statistical power analysis 688 program for the social, behavioral, and biomedical sciences. Behav Res Methods. 2007;39(2):17568991.

690 50. Buscemi S, Verga S, Tranchina M, Cottone S, Cerasola G. Effects of hypocaloric very-low691 carbohydrate diet vs. Mediterranean diet on endothelial function in obese women. Eur J Clin Invest. $692 \quad 2009 ; 39(5): 339-47$.

693 51. Schiavo L, Scalera G, Sergio R, De Sena G, Pilone V, Barbarisi A. Clinical impact of 694 Mediterranean-enriched-protein diet on liver size, visceral fat, fat mass, and fat-free mass in patients 695 undergoing sleeve gastrectomy. Surg Obes Relat Dis. 2015;11(5):1164-70. 
52. Calder P, Harvey D, Pond C, Newsholme E. Site-specific differences in the fatty acid 697 composition of human adipose tissue. Lipids. 1992;27(9):716-20.

698 53. Vissers D, Hens W, Taeymans J, Baeyens J-P, Poortmans J, Van Gaal L. The Effect of Exercise 699 on Visceral Adipose Tissue in Overweight Adults: A Systematic Review and Meta-Analysis. PLoS $700 \quad$ One. $2013 ; 8(2):$ e56415.

701 54. Walther C, Möbius-Winkler S, Linke A, Bruegel M, Thiery J, Schuler G, et al. Regular exercise 702 training compared with percutaneous intervention leads to a reduction of inflammatory markers and 703 cardiovascular events in patients with coronary artery disease. Eur J Cardiovasc Prev Rehabil. $704 \quad 2008 ; 15(1): 107-12$.

705 55. Mozaffarian D. Food and weight gain: time to end our fear of fat. Lancet Diabetes Endocrinol. $706 \quad 2016 ; 4(8): 633-5$.

707 56. Piers L, Walker K, Stoney R, Soares M, O'dea K. The influence of the type of dietary fat on 708 postprandial fat oxidation rates: monounsaturated (olive oil) vs saturated fat (cream). Int J Obes. $709 \quad 2002 ; 26(6): 814-21$.

710 57. Guebre-Egziabher F, Rabasa-Lhoret R, Bonnet F, Bastard J, Desage M, Skilton M, et al. 711 Nutritional intervention to reduce the $n-6 / n-3$ fatty acid ratio increases adiponectin concentration 712 and fatty acid oxidation in healthy subjects. Eur J Clin Nutr. 2008;62(11):1287-93.

713 58. Esposito K, Pontillo A, Di Palo C, Giugliano G, Masella M, Marfella R, et al. Effect of weight 714 loss and lifestyle changes on vascular inflammatory markers in obese women: a randomized trial. 715 JAMA. 2003;289(14):1799-804. 
59. Richard C, Couture P, Desroches S, Lamarche B. Effect of the Mediterranean diet with and

717 without weight loss on markers of inflammation in men with metabolic syndrome. Obesity. $718 \quad 2013 ; 21(1): 51-7$.

719 60. Richard C, Royer M-M, Couture P, Cianflone K, Rezvani R, Desroches S, et al. Effect of the 720 Mediterranean diet on plasma adipokine concentrations in men with metabolic syndrome. $721 \quad$ Metabolism. 2013;62(12):1803-10.

722 61. Blüher M, Rudich A, Klöting N, Golan R, Henkin Y, Rubin E, et al. Two patterns of adipokine 723 and other biomarker dynamics in a long-term weight loss intervention. Diabetes Care. $724 \quad 2012 ; 35(2): 342-9$.

725 62. Hagfors L, Leanderson P, Sköldstam L, Andersson J, Johansson G. Antioxidant intake, plasma 726 antioxidants and oxidative stress in a randomized, controlled, parallel, Mediterranean dietary 727 intervention study on patients with rheumatoid arthritis. Nutr J. 2003;2(1):5.

728 63. George ES, Marshall S, Mayr HL, Trakman GL, Tatucu-Babet OA, Lassemillante A-CM, et al. 729 The effect of high-polyphenol extra virgin olive oil on cardiovascular risk factors: A systematic 730 review and meta-analysis. Crit Rev Food Sci Nutr. 2018:1-24.

731 64. Chew DP, French J, Briffa TG, Hammett CJ, Ellis CJ, Ranasinghe I, et al. Acute coronary 732 syndrome care across Australia and New Zealand: the SNAPSHOT ACS study. Med J Aust. $733 \quad 2013 ; 199(3): 185-91$. 